Proceedings of the XXIII Conference on Applied Crystallography, Krynica Zdrój, Poland, September 20-24, 2015

\title{
Analysis of CMSX-4 Single-Crystalline Turbine Blades Root by Electron and X-Ray Diffraction Methods
}

 \\ ${ }^{a}$ Institute of Materials Science, University of Silesia, 75 Pułku Piechoty 1A, 41-500 Chorzów, Poland \\ ${ }^{b}$ Department of Materials Science, Rzeszów University of Technology, W. Pola 2, 35-959 Rzeszów, Poland
}

The root of single-crystalline turbine blade made of CMSX-4 superalloy were studied. The studied blade was produced by the Bridgman technique in industrial ALD furnace at withdrawal rate of $3 \mathrm{~mm} / \mathrm{min}$. The samples for investigations were cut from the blade root parallel to the withdrawal direction. Metallographic sections of longitudinal samples planes were prepared for further investigations. The samples were analysed using scanning electron microscopy and the Laue diffraction studies. The crystal orientations in macro-scale were determined by analysis of the Laue pattern and local crystal orientations were studied by electron backscattered diffraction technique. Morphology of dendrites were examined by analysis of scanning electron microscopy macro-images. Study of subgrain structure was performed by X-ray diffraction topography. The sharp parallel contrast bands, visible on the X-ray topograms, were related with dendrite cores, arranged with the same direction. Additionally, the low angle boundaries were formed in certain samples, visible on the topograms as contrast shifts. Step changes of local crystal orientation in certain areas were observed on the electron backscattered diffraction maps. The electron backscattered diffraction crystal orientation maps were related to the misorientation visualized in topograms.

DOI: 10.12693/APhysPolA.130.1104

PACS/topics: 81.10.Aj, 61.72.Ff, 61.05.J-

\section{Introduction}

The single-crystalline CMSX-4 superalloy is frequently used in many industry sectors, inter alia, for the production of blades for gas turbines or aircraft engines. The turbine blades are subjected to high loads under high temperatures during operation and therefore they must possess good structural quality and high strength properties. The CMSX-4 nickel-based superalloy possesses required properties, especially heat and creep resistance at high operating temperatures under heavy multiaxial load, acting during operation [1-3]. Due to features of the dendritic structure, complex chemical composition of the CMSX-4 superalloy and shape of the blades, the high probability of casting defects formation exists. The blades geometry can affect the dendritic structure and simultaneously the crystal orientation, which is an important factor influencing the strength of the blades $[4,5]$. Generated structural defects are related to local changes of crystal orientation [6]. The real structure analysis of as cast blades allows to determine the possible changes in obtaining parameters, for preventing the defects creation and improving the structure quality.

The aim of the study was to analyse the crystal orientation and structural defects of as cast single-crystalline blade root, using scanning electron microscope (SEM) technique, electron backscatter diffraction (EBSD) and X-ray topography.

\section{Material and investigations}

The blade was produced by the Bridgman technique using industrial ALD furnace in Research and Develop-

\footnotetext{
*corresponding author; e-mail: jacek.krawczyk@us.edu.pl
}

ment Laboratory for Aerospace Materials, Rzeszów University of Technology. Turbine blade, produced at withdrawal rate of $3 \mathrm{~mm} / \mathrm{min}$ was analysed. The samples for investigations were cut from blade root, parallel to the vertical withdrawal direction G (Fig. 1). The metallographic sections were prepared for further investigations in vertical (longitudinal) planes of samples (P, Fig. 1).



Fig. 1. Scheme of analysed blades with cut sample location.

In order to analyse the dendritic structure of the studied samples SEM observations were performed. SEM macro-images were obtained by stitching several images of microstructure. Morphology of dendrites in analysed areas were examined. The Laue method was used for determination of crystal orientation in macro-scale. The JEOL JSM-6480 scanning electron microscope, equipped with backscattered electron diffraction detector, was used 
for local crystal orientation analysis by EBSD technique. The analysis of subgrain structure was performed by X-ray diffraction topography, using PANalytical microfocus source ( $\mathrm{Cu} K_{\alpha}$ radiation), coupled with an Auleytner camera.

\section{Results and discussion}

Figure 2 shows typical structure of as cast CMSX-4 superalloy in longitudinal cross-section. There are visible three regions containing dendrites with co-directional cores (direction $\mathrm{I}_{1} \mathrm{I}_{2}$ and $\mathrm{I}_{3}$ ), inclined to the direction $\mathrm{G}$ at an $\delta_{1}, \delta_{2}$ or $\delta_{3}$ angle (Fig. 2). Determined angle values are: $\delta_{1}=2.7^{\circ}, \delta_{2}=2.4^{\circ}$, and $\delta_{3}=3.2^{\circ}$. The centre of analysed surfaces is situated above the blade selector and marked as SLR (selector) area (Fig. 2). In SLR area the dendrites cores grow directly from the selector along direction $\mathrm{I}_{2}$. The dendrites with evenly spaced arms have regular shape and fixed size. Excepting the SLR area, the dendrite cores, visible in Fig. 2, form tertiary arms. At the final part of the selector, where the blades root begins to crystallize, secondary dendrite arms grow almost horizontally toward the mould sidewall, forming a base for tertiary dendrite arms growth. There are irregularities in arrangement of dendrites and differences in the size and shape of its arms in areas except the SLR. Estimated value of $\delta_{1}$ and $\delta_{3}$ angles are higher than estimated value of $\delta_{2}$ angle.



Fig. 2. Example of SEM macro-image fragment, obtained from plane P. SLR - selector area, ASBR - approximate subgrain boundary regime, $\mathrm{G}$ - withdrawal direction, $\mathrm{I}_{1}, \mathrm{I}_{2}, \mathrm{I}_{3}$ - directions of dendrite cores, PDA - primary dendrite arms, SDA - secondary dendrite arms, TDA - ternary dendrite arms.
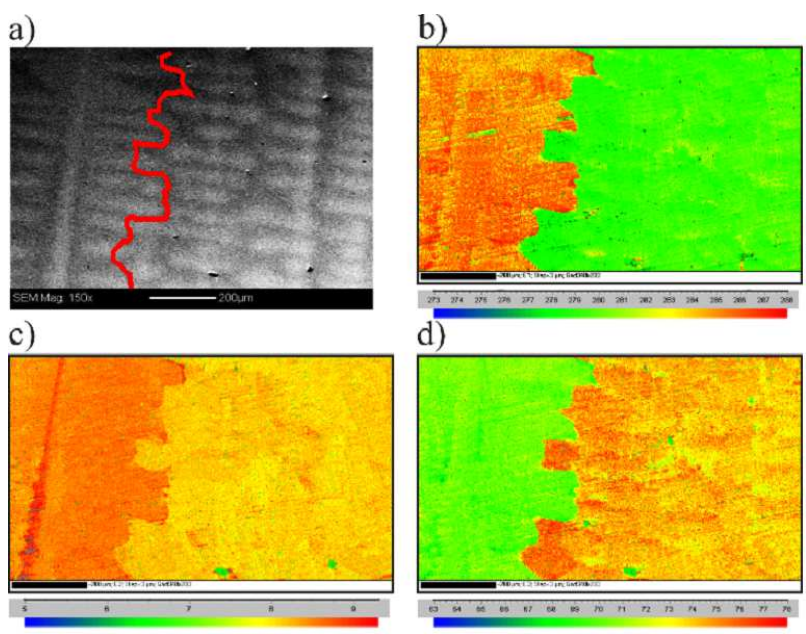

Fig. 3. SEM images recorded by BSE method of the area A (Fig. 2) prepared for EBSD analysis (a) and typical orientation maps for Euler 1 (b), Euler 2 (c) and Euler $3(\mathrm{~d})$. a)

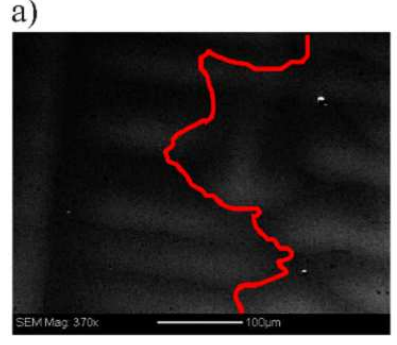

c)



b)



d)

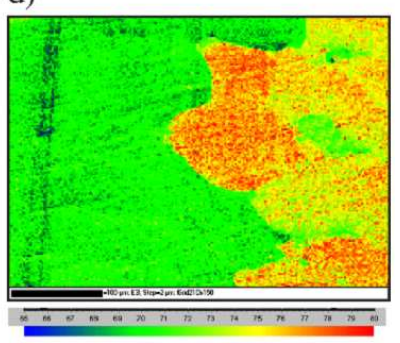

Fig. 4. SEM images recorded by BSE method of the part of area A (Fig. 2) prepared for EBSD analysis (a) and typical orientation maps for Euler 1 (b), Euler 2 (c) and Euler 3 (d).

Figures 3 and 4 show example of EBSD orientation maps and SEM images of the analysed areas. Several dendrites are visible in Fig. 3a which were covered by the EBSD analysis area (A - Fig. 2) near the ASBR. The different colours on the orientation maps correspond to the different crystal orientation of areas in analysed region. The boundary of different crystal orientation areas (red line - Fig. 3a) is irregular and arranged approximately along the dendrite cores. Similar maps and SEM image were obtained at a higher magnification (Fig. 4). Analysed area contains fragments of two neighbouring 
dendrites (Fig. 4a). The boundary line is irregular and passes through all visible component phases. Maximal angle of crystal misorientation of all analysed samples is about $5^{\circ}$.

Crystal misorientation was stated by analysis of the Laue patterns, obtained for diffraction from different areas. The Laue spots arrangement is different for particular analysed areas.

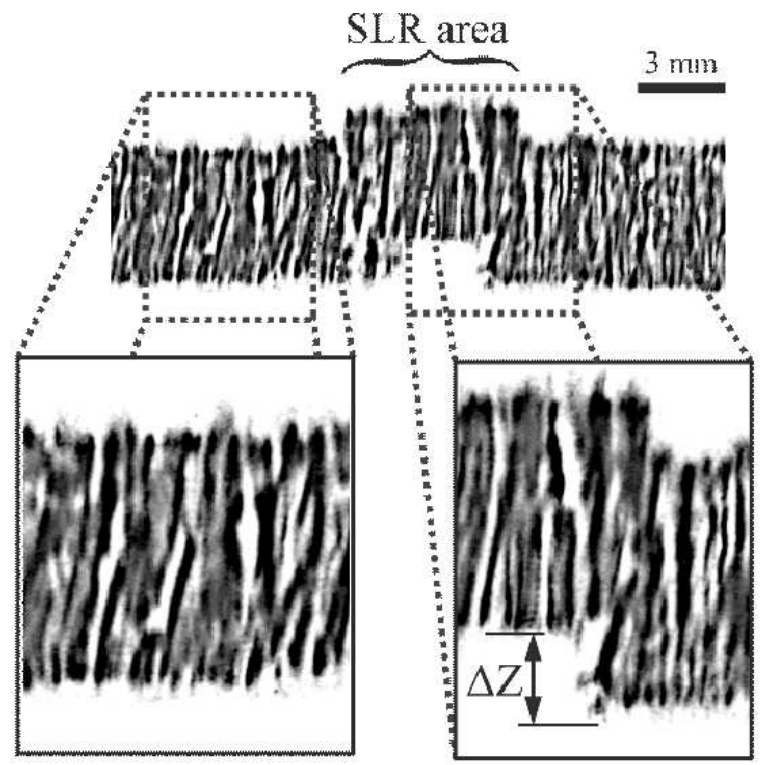

Fig. 5. Typical X-ray topogram obtained with sample oscillation. 220 reflection, $\mathrm{Cu} K_{\alpha}$ radiation, $\Delta Z$ - shift that describes subgrain misorientation.

Figure 5 shows typical X-ray topogram, obtained from P-type plane (Fig. 2). There are visible three main separate contrast areas with different mutual arrangement. Central area of the presented topogram corresponds to selector area (SLR area - Fig. 2). The contrast bands, visible on the topogram are arranged in particular areas parallel to directions $I_{1}, I_{2}$ and $I_{3}$ (Fig. 2), respectively. There are visible shifts between neighbouring contrast areas, exemplary selected and marked on the topogram as $\Delta Z$ (Fig. 5 , right inset). $\Delta Z$ value describes subgrain misorientation angle [7]. Misorientation angle, defined for presented topogram is about $3^{\circ}$. Similar $\Delta Z$ shifts occur at all obtained topograms. Additionally, the right edge of SLR area is covered by neighbouring contrast area. Contrast on the edges of these areas is higher than beyond.

The sharp parallel contrast bands, visible on the X-ray topograms, are related to dendrite cores arranged with the same direction. Inclination of dendrite cores to the withdrawal direction in particular areas of the samples is different. The shifts of contrast areas indicate occurrence of low angle boundaries, formed in certain samples. Various crystal orientation in neighbouring sample areas, defined by EBSD analysis, confirm subgrains occurrence. Additionally, EBSD orientation maps allow to determine subgrain boundary trace. The boundary passes through dendrites and interdendritic regions.

\section{Summary}

Analysis of dendrites morphology shows that the dendrites arrangement and morphology in particular areas is different, especially the inclination of cores to the withdrawal direction. Differences in dendrites arrangement are caused by growth disturbance during initial stage of blades obtaining. The disturbance progresses result in creation of crystal subgrains.

The subgrains appearance and location were analysed by X-ray topography. Low angle boundaries were visualised on the topograms and misorientation angle were defined. Areas of dendrites inclination changes, visualized on SEM macro-images were correlated with changes in contrast on the topograms.

The crystal orientation changes in specific areas were observed on the maps of local crystal orientation, created by EBSD technique. The maps were related to the misorientation, visualized in the X-ray topograms. Slightly differences of misorientation angle may result from possibility of determining from topograms only two of orientation components.

Degree of crystal misorientation, defined using EBSD and X-ray topography is comparable and ranges $3-5^{\circ}$. Not all changes in the crystal orientation of the microareas can be visualized by X-ray topography. Local orientation changes can be analysed by the EBSD technique. Those methods are mutual complementary.

\section{References}

[1] R. Reed, The Superalloys: Fundamentals and Application, Cambridge University Press, Cambridge 2006.

[2] M.J. Donachie, S.J. Donachie, Superalloys. A Technical Guide, ASM International, Materials Park (OH) 2002.

[3] J.C. Williams, E.A. Strake, Acta Mater. 51, 5775 (2003).

[4] D. Shi, J. Huang, X. Yang, H. Yu, Int. J. Fatigue 49, 31 (2013).

[5] Z. Wen, H. Mao, Z. Yue, J.B. Wang, J. Mater. Eng. Perform. 23, 372 (2014).

[6] W. Bogdanowicz, R. Albrecht, J. Sieniawski, K. Kubiak, J. Cryst. Growth 401, 418 (2014).

[7] W. Bogdanowicz, Scr. Mater. 37, 6 (1997). 